\title{
PROGRESS IN THE DEVELOPMENT OF SRF CAVITY TUNERS BASED ON MAGNETIC SMART MATERIALS ${ }^{1}$ \\ C. Joshi ${ }^{2}$, A. Pappo, D. Upham, Energen, Inc., Billerica, MA \\ J. Preble, Jefferson Laboratory, Newport News, VA
}

\section{Abstract}

Energen, Inc. has developed and demonstrated an SRF cavity tuning mechanism based on magnetic smart materials. Magnetic "smart" materials change their shape in a reversible and repeatable manner when exposed to a small magnetic field. A fine-tuning mechanism with a 2 $\mathrm{kHz}$ tuning range on a nominal resonant frequency of $1.497 \mathrm{GHz}$. was successfully demonstrated in 1999 [1]. Since then, Energen has been developing a tuning mechanism based on its linear stepper motors. These stepper motors are designed to deliver high-force precision linear motion of tens of millimeters at cryogenic temperatures. A locking mechanism built into the stepper motor enables the tuner to be locked into position when the power is turned off. This new tuning technology will eliminate the mechanical feeds through the vacuum jacket and reduce the complexity of the cryostat design and assembly. Performance and capabilities of a prototype SRF cavity tuner will be reported.

\section{INTRODUCTION}

\subsection{Jefferson Laboratory Accelerator}

The Jefferson Laboratory of Newport News, VA operates the largest continuous beam electron accelerator in the world. Approximately 344 superconducting RF cavities operate at a resonant frequency of $1.497 \mathrm{GHz}$ to accelerate the electrons as they pass through each cavity. These cavities are designed and built to exacting geometric tolerances. However, precise adjustments must be made to the length of each SRF cavity in order to finetune each cavity and synchronize their resonance frequencies during accelerator operation.

Jefferson Laboratory uses a mechanical system as shown in Figure 1. The system uses a rotating stepper motor at room temperature to drive a wormwheel gear reduction connected to a ball screw shaft. As the shaft turns, the two cell holders are squeezed together compressing the cavity and changing its resonant frequency. This mechanical system is relatively simple in concept; however, it is difficult to use for accurate cavity tuning. In operation, this system, like any other mechanical assembly, has backlash. In addition to the elastic behavior of the components, split shaft couplers are used for the rotating feed through to allow for thermal contraction during cool down. This split assembly adds to the inherent backlash of the system.

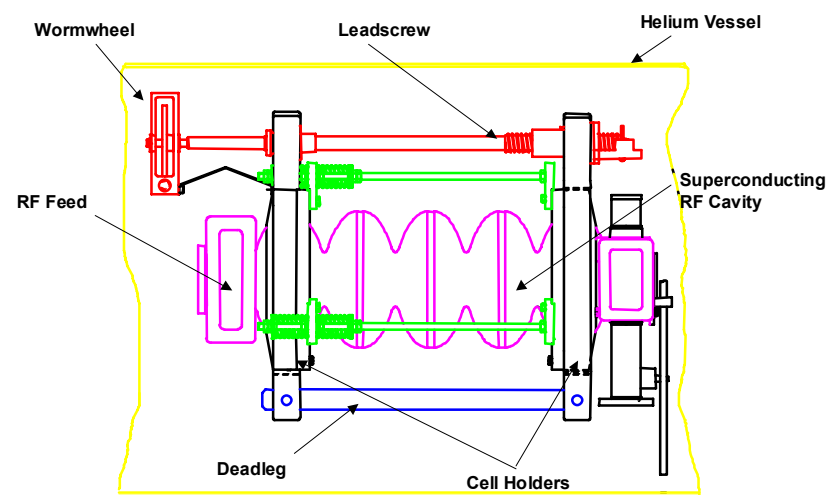

Figure 1 - The CEBAF superconducting RF cavity showing its mechanical tuner.

\section{MAGNETIC "SMART" MATERIALS}

Magnetic "smart" materials (MSM) change their shape (elongate) in a predictable and reversible manner when exposed to a magnetic field. In these ferromagnetic materials, an applied magnetic field causes rotation of the magnetization towards the field direction within domains and/or motion of the domain walls to increase the size of the domains with magnetization vectors close in direction to the applied field. When the magnetization is completely aligned, saturation occurs. Increasing the applied magnetic field beyond this point produces no further elongation. This behavior only occurs in a material at temperatures below its Curie temperature. The amount of elongation at saturation is the most fundamental measure of a magnetostrictive material. Table 1 shows a compilation of some materials and the maximum elongation they exhibit.

Table 1 -- Saturation strain and Curie temperatures of selected materials [2].

\begin{tabular}{l|cc}
\multicolumn{1}{c|}{ Material } & $\begin{array}{c}\text { Max. Elongation } \\
\left(\times 1^{-6}\right)\end{array}$ & $\begin{array}{c}\text { Curie } \\
\text { Temperature } \\
(\mathrm{K})\end{array}$ \\
\hline $\mathrm{Ni}$ & -50 & 630 \\
$\mathrm{Fe}$ & -14 & 1040 \\
$\mathrm{SmFe}$ & -2340 & 690 \\
$\mathrm{Fe}_{3} \mathrm{O}_{4}$ & 60 & 860 \\
$\mathrm{DyFe}_{2}$ & 650 & 630 \\
$\mathrm{TbFe}_{2}$ & 2630 & 700 \\
$\mathrm{~Tb}_{0.3} \mathrm{Dy}_{0.7} \mathrm{Fe}_{1.9}(\mathrm{Terfenol-D)}$ & $1600-2400$ & 650 \\
$\mathrm{~Tb}_{0.6} \mathrm{Dy}_{0.4} @ 77 \mathrm{~K}$ & 6300 & 210 \\
$\mathrm{~Tb}_{0.5} \mathrm{Zn}_{0.5}$ & 5500 & 180 \\
$\mathrm{~Tb}_{1-\mathrm{x}} \mathrm{Dy}_{\mathrm{x}} \mathrm{Zn}$ & 5000 & 200 \\
\hline
\end{tabular}

\footnotetext{
${ }^{1}$ Supported by the Nuclear Physics Division of the U. S. Dept. of Energy through the SBIR Program

2 E-mail: chad@EnergenInc.com
} 
Note that the materials exhibiting the highest magnetostrictive strain have Curie temperatures below room temperature. These materials show great promise for cryogenic actuator applications such as the SRF cavity tuners. Typical performance curve for a rod of TbDyZn is shown in Figure 2.

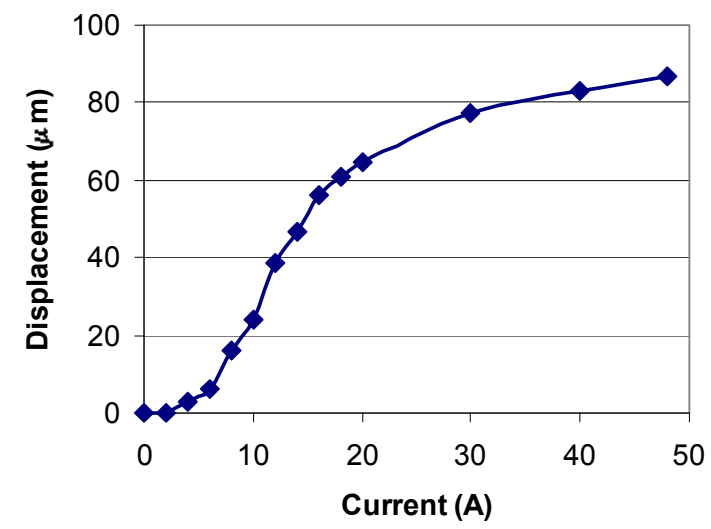

Figure 2 - Magnetostriction of a TbDyZn alloy at $4.2 \mathrm{~K}$ shows high saturation strain.

\subsection{Actuators}

An MSM-based actuator consists of a rod of MSM surrounded by a coil. When current passes through the coil, the rod elongates and the motion can be used to do useful work. Energen, Inc. offers several models of actuators for precision positioning motion up to $300 \mu \mathrm{m}$ as shown in Figure 3. These devices are ideal for applications where short stroke and high force and rapid response are needed. The response time for these actuators to move their full stroke ranges from $25 \mu$ s to $300 \mu \mathrm{s}$. Complete specifications for these actuator are available on our website [4].

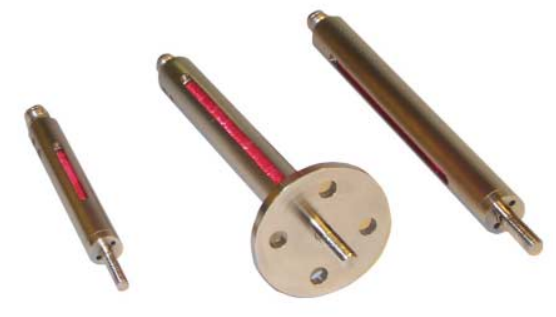

Figure 3 - Typical actuators offered by Energen, Inc.

\subsection{Linear Motors}

Energen has developed a linear motor capable of long range motion. The stepper motor actuator consists of a shaft held by a pair of clamps. The shaft consists of a linear actuator with a connecting rod extending from each end. Energizing the MSM actuator contained within each clamp causes it to release its hold on the connecting rod allowing it to move axially. A photograph of two Energen stepper motors is shown in Figure 4. Detailed information about the stepper motor operation has been presented previously [2].

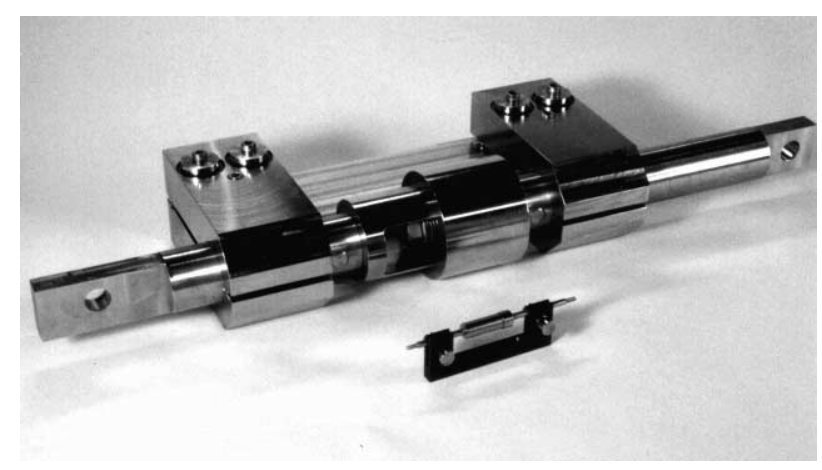

Figure 4 - Two different sizes of linear motor illustrate the range of products available.

\subsection{Control Electronics}

The control electronics for the linear stepper motor enables a wide range of motor operational flexibility through a digital state control circuit. In addition to setting the operating mode (stepper or fine tune), the direction of motion, the step size, and stepping frequency can be adjusted. More recently, a computer interface for the motor controller has been developed. This interface enables computerized operation of the stepper motor through either stand-alone application software or using subroutines callable from C, Visual Basic or Labview programs. The supported communications protocols between the computer and the controller are RS-232C, RS-422/485 and USB.

\section{CAVITY TUNER}

\subsection{Cavity Interface}

Under a Cooperative R\&D Agreement (CRADA) between Energen and Jefferson Laboratory, Energen obtained a 5-cell SRF cavity from Jefferson Laboratory for the testing of the tuner. The Energen tuner was designed to be a replacement for the mechanical tuner that is currently used providing the added benefit of better tuning resolution.

A drawing of the tuner connected to the cavity is shown in Figure 5 below. The end cells of the cavity are attached to a cell holder. The inactive cell holder (shown on the right side) is rigidly connected to the end plate by four connecting rods. The active cell holder (shown on the left side) is free to move axially. The end plate and the active cell holder are connected to a lever arm by stainless steel wire ropes. The linear stepper motor is mounted on a platform beside the SRF cavity. When activated it pushes a rolling contact against the lever arm. The rotation of the lever arm pulls the active cell towards the endplate stretching the cavity along its axis thereby changing its resonant frequency. When retracted the stepper motor can be mechanically disengaged from the tuner system, 
separating the rolling contact from the lever arm. This feature prevents unwanted stress build up that may occur during cool down.

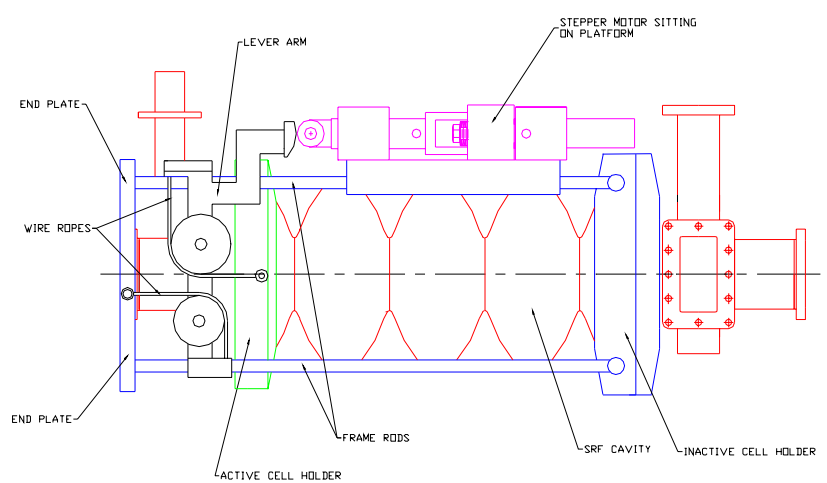

Figure 5 - Geometry of the Energen SRF cavity tuner.

\subsection{Advantages}

This type of tuning system has several distinct advantages, it:

- Eliminates sliding or rotating penetrations through the vacuum cryostat

- Simplifies cryostat design and assembly

- Delivers both coarse and fine tuning in a single device

- Can be used for fast tuning (microphonics)

- Locks in position when powered off

\subsection{Mechanical Testing}

Diagnostic testing is relatively easy because the stepper motor operates at temperatures as high as $150 \mathrm{~K}$. Energen has been testing the mechanical performance of the SRF cavity tuner at liquid nitrogen temperatures at our facilities. Figure 7 is a photograph of the five cell cavity and tuner assembled and tested at Energen's facilities. The tuner assembly is designed to extend the cavity a total of $3 \mathrm{~mm}$ in length corresponding to a dynamic tuning range of $300 \mathrm{kHz}$. Our preliminary tests have been conducted over a tuning range of $1.8 \mathrm{~mm}$. Further scheduled testing will extend this range.

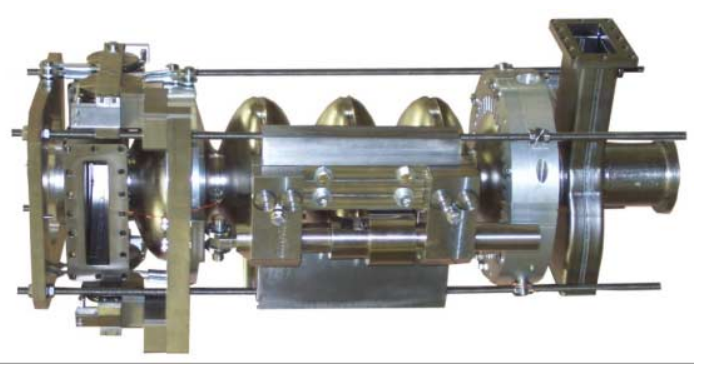

Figure 7 - SRF Cavity with Tuner
Figure 8 shows data from the first test of the mechanical movement of the tuner system. The elongation of the cavity is shown as a function of the movement of the linear motor showing that the lever provides a mechanical advantage of almost 5 to 1 .

The SRF cavity and tuner are presently being prepared for frequency testing. The cavity will be sealed and evacuated. A RF injection port and antenna at opposite ends of the cavity will enable us to measure the resonant frequency.

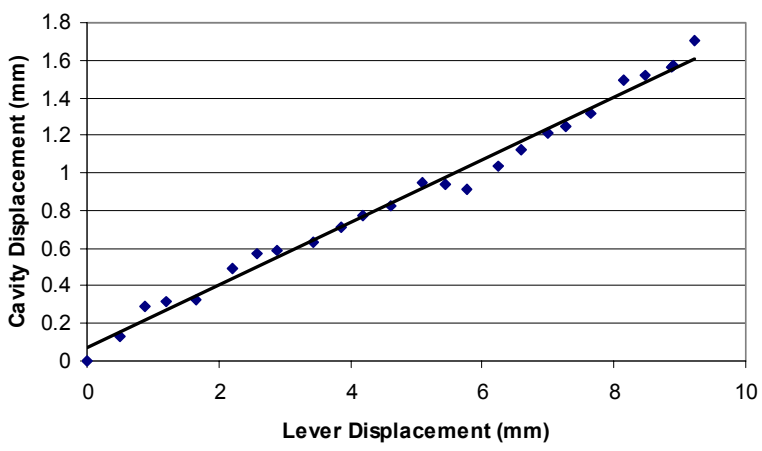

Figure 7 - Mechanical movement of tuning system

\section{CONCLUSIONS}

Energen, Inc. has designed and built a tuning mechanism for superconducting radio frequency (SRF) cavities used in particle accelerators. The prototype is designed for a five-cell cavity, but can be easily adapted to other cavity configurations. Preliminary tests demonstrate cavity elongation of $1.8 \mathrm{~mm}$ corresponding to a tuning range of $180 \mathrm{kHz}$ at a nominal resonant frequency of $1.497 \mathrm{GHz}$.

\section{ACKNOWLEDGEMENTS}

We would like to thank T. Grimm of the National Superconducting Cyclotron Laboratory, E. Lansing, MI for his advice and guidance with the cavity testing.

\section{REFERENCES}

[1] Joshi, et al, Particle Accelerator Conference 1999, Paper No. 931.

[2] Magnetostrictive Materials, K. B. Hathaway and A. E. Clark, MRS Bulletin, Vol. XVIII, No. 4, April 1993.

[3] Joshi et al, RF Superconductivity Workshop, Santa Fe, NM, Nov. 1-5, 1999.

[4] http://www.EnergenInc.com 